\title{
Coherent motion with linear coupling
}

\author{
A. Burov and V. Lebedev \\ Fermi National Accelerator Laboratory, Batavia, Illinois 60510, USA
}

(Received 18 December 2006; published 18 April 2007)

\begin{abstract}
Coupling of the transverse degrees of freedom modifies analysis of beam coherent motion. A general, simple, and effective rule to do that is derived.
\end{abstract}

DOI: 10.1103/PhysRevSTAB.10.044402

PACS numbers: 29.27.Bd, 29.20.Dh

\section{INTRODUCTION}

When the two fractional tunes of a storage ring are equal, $\left\{\nu_{x}\right\}=\left\{\nu_{y}\right\}$, the beam stays at a coupling resonance. This line in the tune space is of a special attraction: staying there maximizes tune area free from the dangerous resonances. That is why so many machines stayed, stay, or plan to be there. Near the coupling resonance, even a small skew quadrupole or solenoid may result in a significant change of the beam optics, making it strongly coupled. If so, a conventional uncoupled 2D optical formalism cannot be used; instead, a 4D analysis has to be applied. Thus, any beam issue underlain by the optics has to be revisited, assuming that eigenmodes do not describe planar vertical and horizontal motion any more. One of these issues is a problem of the beam transverse coherent motion. This problem was considered in Refs. [1-3], and discussed in [4]. Here, we suggest our view of the problem, and come to a solution, which is general and simple at the same time.
The leading idea is that the classical mechanics is invariant over the canonical transformations. In a basis of the eigenmodes, the beam motion gets to be uncoupled, and formally similar to the conventional $x-y$ uncoupled case. There is though a single difference between the $x-y$ space and the space of the normal modes. This difference relates to wake functions or impedances, which are given in the $x-y$ space. Thus, to solve the problem, the wakes and impedances have to be properly projected on the eigenvectors.

\section{EIGENMODE PERTURBATIONS}

For arbitrary coupling, the beam optics can be described in terms of $4 \mathrm{D}$ eigenvectors. Hereafter, a parametrization suggested in [5] is used, where the 4 eigenvectors $\mathbf{V}_{1}, \mathbf{V}_{-1} \equiv \mathbf{V}_{1}^{*}, \mathbf{V}_{2}, \mathbf{V}_{-2} \equiv \mathbf{V}_{2}^{*}$ of a revolution matrix $\mathbf{R}$ are presented as follows:

$$
\begin{aligned}
& \mathbf{V}_{1}=\left(\sqrt{\beta_{1 x}},-\frac{i(1-u)+\alpha_{1 x}}{\sqrt{\beta_{1 x}}}, \sqrt{\beta_{1 y}} e^{i \nu_{1}},-\frac{i u+\alpha_{1 y}}{\sqrt{\beta_{1 y}}} e^{i \nu_{1}}\right)^{\mathrm{T}} ; \\
& \mathbf{V}_{2}=\left(\sqrt{\beta_{2 x}} e^{i \nu_{2}},-\frac{i u+\alpha_{2 x}}{\sqrt{\beta_{2 x}}} e^{i \nu_{2}}, \sqrt{\beta_{2 y}},-\frac{i(1-u)+\alpha_{2 y}}{\sqrt{\beta_{2 y}}}\right)^{\mathrm{T}} ;
\end{aligned}
$$

where the superscript $\mathrm{T}$ stands for the transposed form, and $\mathbf{R} \cdot \mathbf{V}_{m}^{(0)}=\exp \left(-i \mu_{m}\right) \mathbf{V}_{m}^{(0)}$. Components of the $4 \mathrm{D}$ vectors are transverse coordinates and angles, $\left(x, \theta_{x}, y, \theta_{y}\right)$; in case of nonzero longitudinal magnetic field, the angles are modified according to a conventional rule for the canonical momenta [5]. Eigenvector parameters $\beta_{1 x}, \beta_{2 y}$, etc., are determined by the machine optics. The symplecticity requires then a specific orthogonality

$$
\mathbf{V}_{m}^{+} \cdot \mathbf{U} \cdot \mathbf{V}_{n}=-2 i \delta_{m n} \operatorname{sgn}(m)
$$

with the superscript + meaning Hermite conjugation, $\delta_{m n}$ is the Kronecker symbol, $\operatorname{sgn}(m)$ is the sign function, and

$$
\mathbf{U}=\left(\begin{array}{cccc}
0 & 1 & 0 & 0 \\
-1 & 0 & 0 & 0 \\
0 & 0 & 0 & 1 \\
0 & 0 & -1 & 0
\end{array}\right)
$$

is the symplectic unit matrix. This formalism is in fact an extension of the Ripken-Mais presentation [6], and is closely related to the Edwards-Teng parametrization [7]. Any vector $\mathbf{X}$ in the $4 \mathrm{D}$ phase space can be expanded over the eigenvectors (1):

$$
\begin{gathered}
\mathbf{X}=\sum_{n} C_{n} \mathbf{V}_{n} ; \quad C_{n}=\frac{i}{2} \mathbf{V}_{n}^{+} \cdot \mathbf{U} \cdot \mathbf{X} ; \\
C_{-n}=C_{n}^{*} ; \quad(n>0) .
\end{gathered}
$$

Now, an elementary act of two-particle interaction has to be considered in terms of the eigenmodes. Following Chao's notations [8], the elementary kick for angles of the following particle $\left(\Delta \theta_{x}, \Delta \theta_{y}\right)$ is expressed as

$$
\Delta \theta_{x}=-e^{2} x W_{x} /\left(p_{0} v_{0}\right) ; \quad \Delta \theta_{y}=-e^{2} y W_{y} /\left(p_{0} v_{0}\right)
$$


Here $e$ is the particle charge, $p_{0}$ and $v_{0}$ are a longitudinal velocity and momentum in the laboratory frame, $x$ and $y$ are the offsets, and $W_{x, y}$ are the wake functions. In terms of the $4 \mathrm{D}$ vector $\mathbf{X}=\left(x, \theta_{x}, y, \theta_{y}\right)$, this can be expressed as a perturbation $\Delta \mathbf{X}=\mathbf{W} \cdot \mathbf{X}$ with the wake matrix elements $\mathbf{W}_{2,1}=-e^{2} W_{x} /\left(p_{0} \boldsymbol{v}_{0}\right), \mathbf{W}_{4,3}=-e^{2} W_{y} /\left(p_{0} \boldsymbol{v}_{0}\right)$, and zeros for all other matrix elements. In terms of the complex amplitudes $C_{n}(5)$, this kick is expressed as

$$
\begin{aligned}
\Delta C_{n} & =\frac{i}{2} \mathbf{V}_{n}^{+} \cdot \mathbf{U} \cdot \Delta \mathbf{X}=\frac{i}{2} \sum_{m} \mathbf{V}_{n}^{+} \cdot \mathbf{U} \cdot \mathbf{W} \cdot \mathbf{V}_{m} C_{m} \\
& \equiv \frac{i}{2} \sum_{m} \mathbf{G}_{n, m} C_{m} .
\end{aligned}
$$

The kick matrix $\mathbf{G}$ is not diagonal generally; so, when the mode $m$ is originally excited, the wake drives other modes $n \neq m$ as well. However, when the wake is small enough, it can be treated as a small perturbation of the coherent eigenmode amplitudes. In this case, in the first order of the perturbation theory, only diagonal elements of the perturbation are important, similar to the quantum mechanics (see [9] for more details). The wake mixing can be considered as small in this sense, when the tune separation of the two transverse modes is much bigger than the wakedriven coherent tune shift:

$$
\left|\nu_{1}-\nu_{2}\right| \gg \Delta \nu_{\text {coh }}
$$

In reality, this condition is typically satisfied. If it is not, nondiagonal elements of the kick matrix $\mathbf{G}$ have to be taken into account as well, leading to some modification of results. Below, the condition (8) is assumed satisfied, so the perturbation formalism is approved. Thus, only diagonal elements in Eq. (7) count; they are calculated as follows:

$$
\begin{gathered}
G_{n} \equiv \mathbf{G}_{n, n}=\mathbf{G}_{n,-n}=-\frac{e^{2}}{p_{0} \boldsymbol{v}_{0}}\left(W_{x} \beta_{n x}+W_{y} \beta_{n y}\right) ; \\
(n=1,2) .
\end{gathered}
$$

This result already shows how the wake is projected on the eigenmodes. However, one more step may be useful for understanding. The complex amplitudes $C_{n}$ can be presented with explicitly written real and imaginary parts as

$$
C_{n}=\frac{q_{n}}{2}+i \frac{p_{n}}{2} ; \quad(n=1,2) .
$$

It is straightforward to show that a linear phase space transformation from the original variables $\left(x, \theta_{x}, y, \theta_{y}\right)$ to the new variables $\left(q_{1}, p_{1}, q_{2}, p_{2}\right)$ is canonical, since they are related to each other by a symplectic matrix, composed from real and imaginary parts of the eigenvectors $\mathbf{V}$ (see Ref. [5]). Thus, $q_{1}, q_{2}$ are new canonical coordinates, and $p_{1}, p_{2}$ are the corresponding canonical momenta. It follows then that a single excited mode gets the wake-driven kick with

$$
\begin{aligned}
\Delta q_{n}= & 0 \\
\Delta p_{n}= & G_{n} q_{n}=-\frac{e^{2}}{p_{0} v_{0}}\left(W_{x} \beta_{n x}+W_{y} \beta_{n y}\right) q_{n} ; \\
& (n=1,2) .
\end{aligned}
$$

Equations (11) show how canonical momentum is perturbed by a small localized wake. Having that, the Vlasov equation with all its results in the phase space $\left(q_{1}, p_{1}\right)$ are exactly identical to the uncoupled case $\left(x, \theta_{x}\right)$, with the following substitution rules for the tune $\nu_{x}=\mu_{x} /(2 \pi)$, wake times beta function $W_{x} \beta_{x}$, and, thus, impedance times beta function $Z_{x} \beta_{x}$ :

$$
\begin{gathered}
\nu_{x} \rightarrow \nu_{1} ; \quad W_{x} \beta_{x} \rightarrow W_{x} \beta_{1 x}+W_{y} \beta_{1 y} ; \\
Z_{x} \beta_{x} \rightarrow Z_{x} \beta_{1 x}+Z_{y} \beta_{1 y} .
\end{gathered}
$$

Note that these rules work both for coasting or bunched beam, and do not depend on a shape of the longitudinal potential well. Any solution of the Vlasov equation for an uncoupled beam can be immediately rewritten to the coupled case with these simple rules. After that, the result looks formally similar, while its practical consequences are generally different because of two reasons. First, the incoherent betatron spectrum is changed by the coupling, $\nu_{x} \rightarrow$ $\nu_{1}$; thus, the Landau damping is changed. This point is missed in Refs. [2,3], where denominators of dispersion integrals are based on the uncoupled incoherent tunes. And second, an amplitude of the coherent shift $\propto Z_{x} \beta_{1 x}+$ $Z_{y} \beta_{1 y}$ is a function of coupling as well. The wake substitution rule (12) is valid both for conventional driving (or dipole) wake, and for the detuning (quadrupole) wake (for more information about the two wakes, see e.g. Ref. [10]).

The substitution rules (12) show disagreement both with results of Ref. [1], and Refs. [2,3].

A head-tail growth rate $\alpha$ was derived in Ref. [1] for a coupled optics within a two-particle model, and the two rates were found to be identical. In a simplified form, cited in Ref. [11], the rate looks like

$$
\alpha \propto \nu_{x}^{\prime} W_{x}+\nu_{y}^{\prime} W_{y},
$$

where $\nu_{x}^{\prime}, \nu_{y}^{\prime}$ are the chromaticities far from the coupling resonance. Applied for the same problem, the substitution rules (12) lead to

$$
\alpha_{n} \propto \nu_{n}^{\prime}\left(\beta_{n x} W_{x}+\beta_{n y} W_{y}\right) .
$$

Clearly, the two results are significantly different. They may become identical only if accidentally $\nu_{x}^{\prime} / \nu_{y}^{\prime}=$ $\beta_{n x} / \beta_{n y}$ both for $n=1$ and $n=2$. Generally, this condition cannot be correct: the left-hand side is determined by sextupoles, while the right-hand side is given by coupled linear optics.

There are two significant disagreements between the rules (12) and Refs. [2,3]. According to these papers, a localized skew-quad entangles coasting beam modes with 
different longitudinal numbers. We cannot agree with that. Indeed, localization of skew quads, as well as normal quads, still preserves the longitudinal wave number, since the growth time is much longer than the revolution time. When the Vlasov equation is averaged over fast variables, resulting equations on the slow growing coherent amplitudes become homogeneous over the ring, so the longitudinal Fourier harmonics are true eigenmodes of the coasting beam. The second disagreement between (12) and Refs. [2,3] is that denominators in dispersive integrals [e.g. Eq. (7) of Ref. [2]] are uncoupled, which excludes correct calculation of Landau damping from those equations.

\section{CONCLUSION}

A method to treat $x-y$ coupling for analysis of beam transverse coherent oscillations is presented. The method effectively reduces a coupled problem to an uncoupled one, making the two problems identical - for any strength of coupling, any sort of bunching, any wake functions, any space charge, etc. Intentionally, any further analysis, like possible use of coupling, is left outside of the scope of this paper.

[1] R. Talman, CBN-97-16, 1997.

[2] E. Metral, Part. Accel. 62, 259 (1999).

[3] R. Cappi, E. Metral, and D. Mohl, Proceedings of EPAC 2000, Vienna, 2000.

[4] G.P. Goderre et al., Proceedings of PAC 1991, San Francisco, 1991.

[5] V. Lebedev and S. Bogacz, JLAB-ACC-99-19, 1999.

[6] F. Willeke and G. Ripken, Methods of Beam Optics, AIP Conf. Proc. No. 184 (AIP, New York, 1989), p. 758.

[7] D. A. Edwards and L. C. Teng, IEEE Trans. Nucl. Sci. 20, 885 (1973).

[8] A.W. Chao, Physics of Collective Beam Instabilities in High Energy Accelerators (Wiley, New York, 1993).

[9] A. Burov, Phys. Rev. ST Accel. Beams 9, 120101 (2006).

[10] A. Burov and V. Danilov, Phys. Rev. Lett. 82, 2286 (1999).

[11] S. Henderson et al., CBN-97-20, 1997. 